\title{
COVID-19 AS A FORM OF HYBRID WAR
}

\author{
MALKHAZ CHIKOBAVA \\ $\mathrm{PhD}$ in Economics, Associate professor, \\ Ivane Javakhishvili Tbilisi State University, Georgia \\ malkhaz.chikobava@tsu.ge
}

Abstract. The article presents a comparative analysis of the Great Depression of the 1930s and the current global financial and economic crisis. It is emphasized that overcoming the first great depression and eliminating imbalances in the economy was achieved only during the Second World War, and the modern crisis, which has been raging for 11 years, has not yet ended. This crisis was not followed by the elimination of all imbalances accumulated in the economy. After the acute phase (recession) ended in 2009, stagnation (depression) occurred, which needed to be restored. We have been waiting for a revival for many years, now 2020 , but it is not visible.

Comparing the crisis of the 30s of the last century and the current global financial and economic crisis, the following differences are obvious: stagnation in the 1930s lasted from 1933 to 1939, or six years that ultimately ended in World War II. After the crisis of 2007-2009, stagnation continued for 11 years, with depression almost two times longer and more delayed. Despite the fact that in a sense, the situation in Western countries is better in the 21st century than in the 1930s, since there is no longer the Soviet Union with its dynamically developing economy, but China has unprecedentedly high rates of economic growth. The thirty-year economic dynamics of China can definitely be called a boom phase. Not a single country in the West has experienced such a long boom in the history of capitalism. It is clear that in such a situation the West must do something.

Of course, the West, through war, has repeatedly overcome the accursed resistance of the capitalist rule of production. But with the help of war, overcoming the contradiction of capitalism today is deadly dangerous. The first two world war occurred without the use of nuclear weapons or any other weapons of mass destruction. The third world war, of course, will be accompanied by the inevitable use of weapons of mass destruction. And therefore, it is necessary to change something in the world war, which will magically help correct the imbalance of the capitalist economy, revive it, and maintain the status quo of the ruling elite.

An alternative to a hot war can be the Cold War, which today they prefer to call hybrid. It involves the use of financial, commercial, economic, psychological and information resources. However, all this is not enough to provide the authorities with powers that would allow them to move from market methods to administrative-command methods of managing the economy. It is with the help of the latter that the imbalance that has accumulated in the economy can be overcome.

It is in this context that Coronavirus "appears" as an alternative to the global war to eliminate the imbalances accumulated in the leading economies of the world.

\section{KEYWORDS: GLOBAL ECONOMIC CRISIS, RECESSION, DEPRESSION, CORONAVIRUS.}

For citation: Chikobava, M., (2020). Covid - 19 as a Form of Hybrid War. Globalization and Business, 10. 79-85. (In Georgian) https://doi.org/10.35945/gb.2020.10.010 


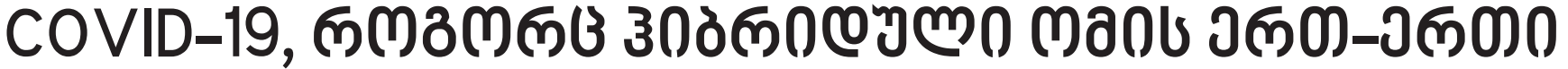 эмळаs}

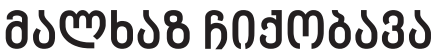

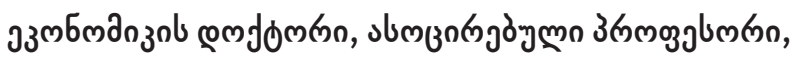

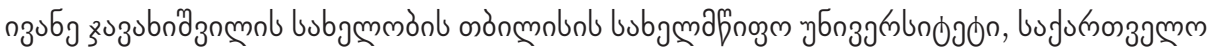

malkhaz.chikobava@tsu.ge

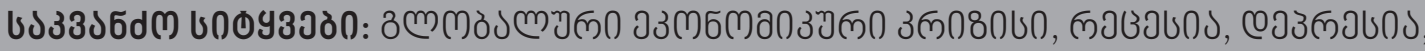

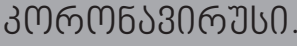

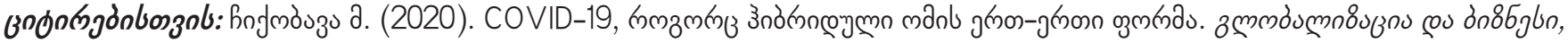
№10, 33. 79-85. https://doi.org/10.35945/gb.2020.10.010

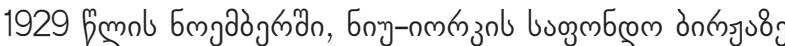

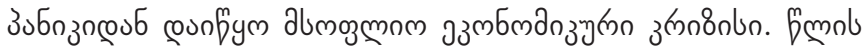

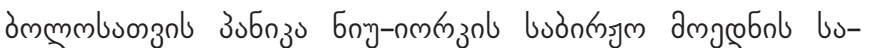

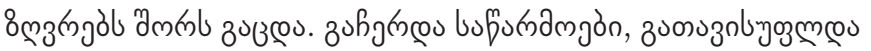

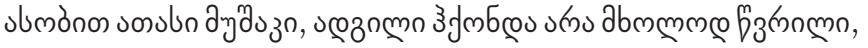

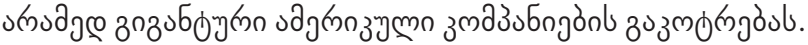

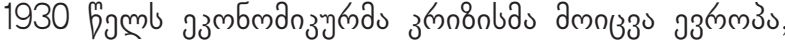

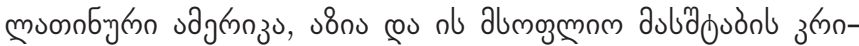

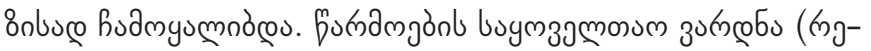

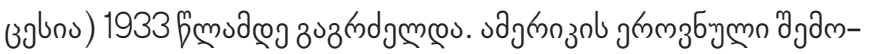

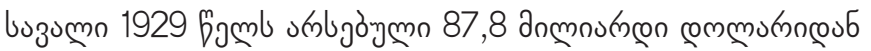

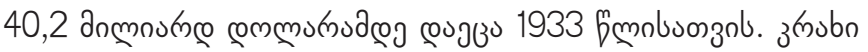

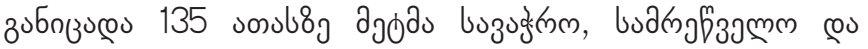
оुnбsбby

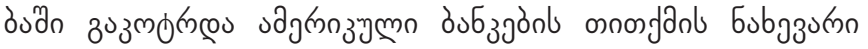
(https://www. fondsk.ru/news/2019/10/24/chernyjchetverg-24-oktjabrja-1929-goda-bankrotstvo-nauchnoobosnovannogo-optimizma-49304.html).

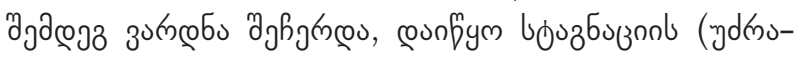

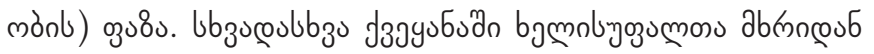

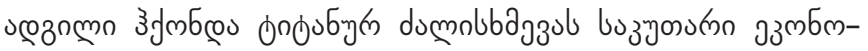

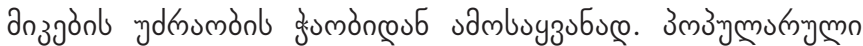

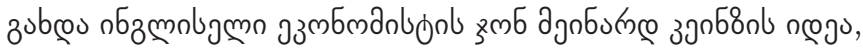

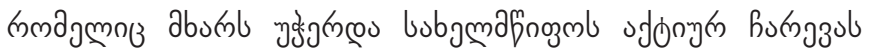

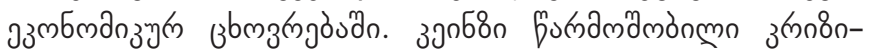

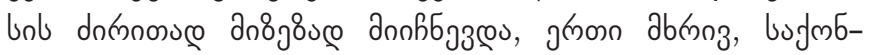

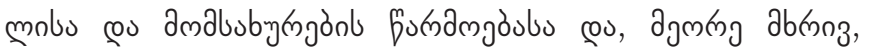

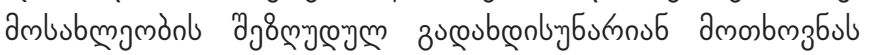

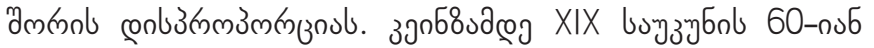

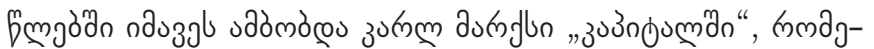

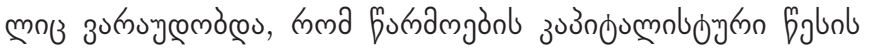

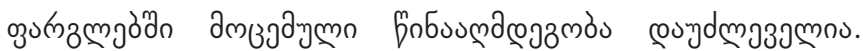

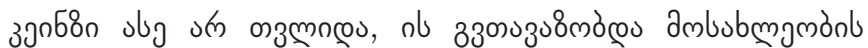

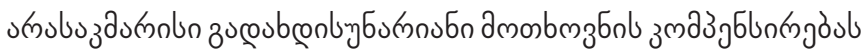

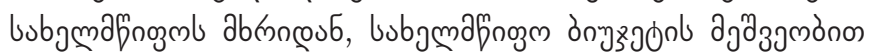

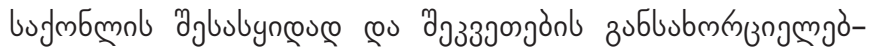

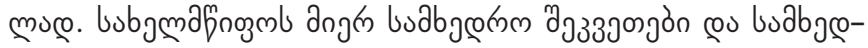

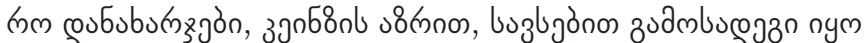

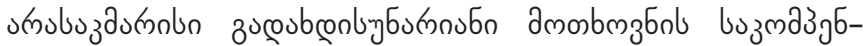

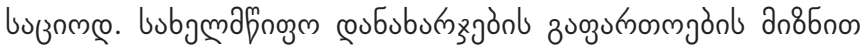

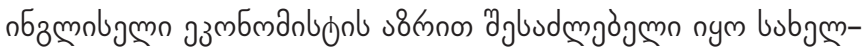

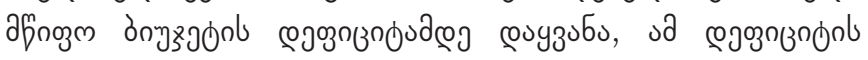

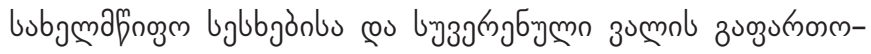

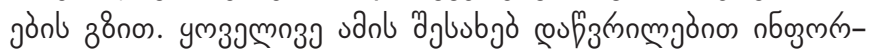

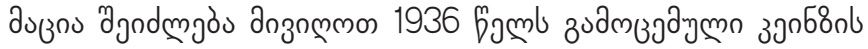

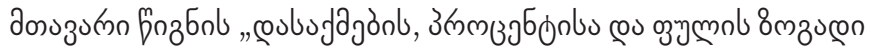

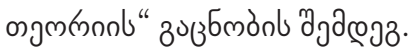

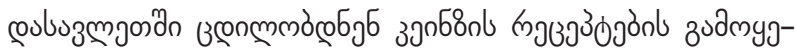

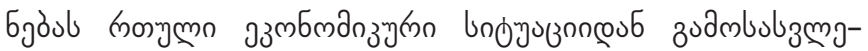

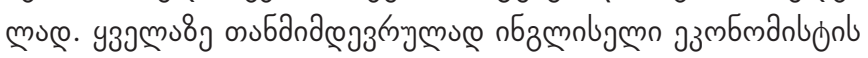

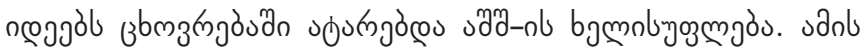

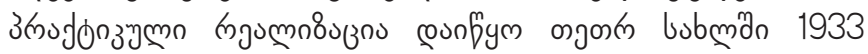

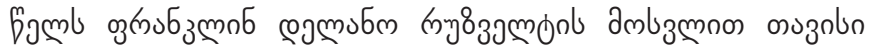

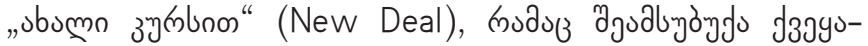

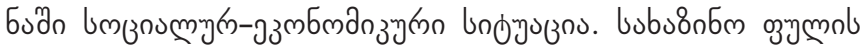

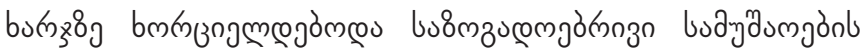

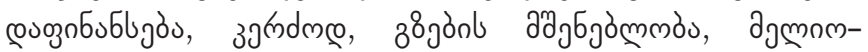

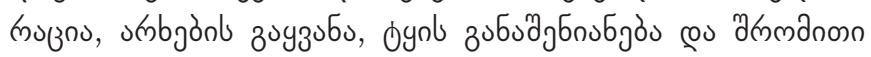

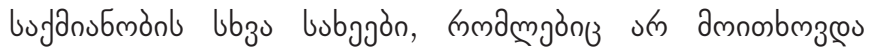

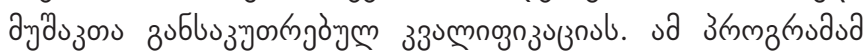

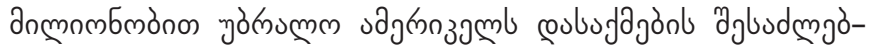

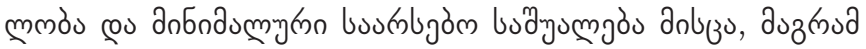

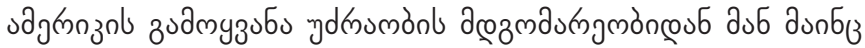

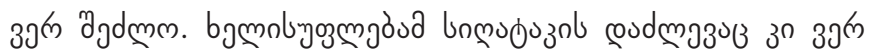




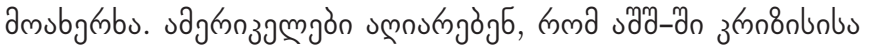

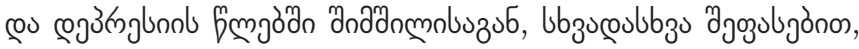

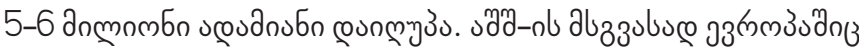

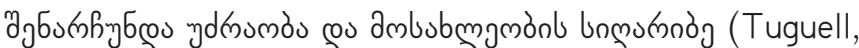
1957).

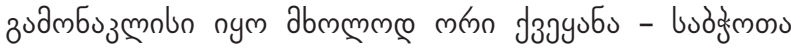

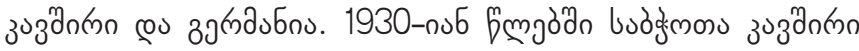

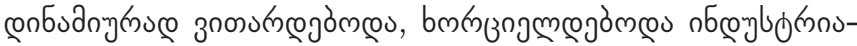

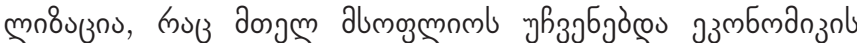

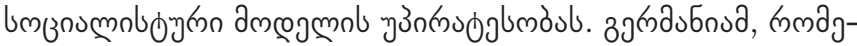

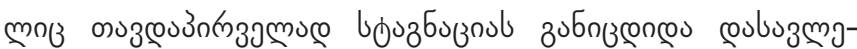

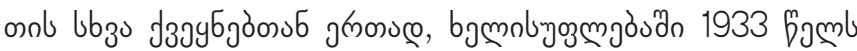

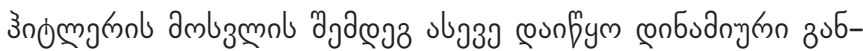

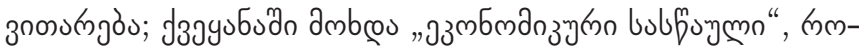

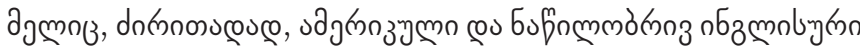

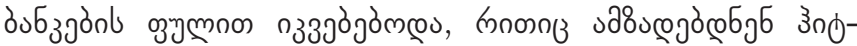

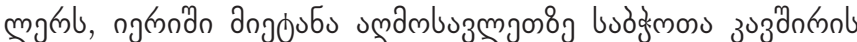

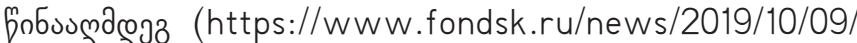
anglo-amerikanskij-biznes-s-nacistami-49197.html)

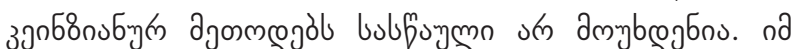

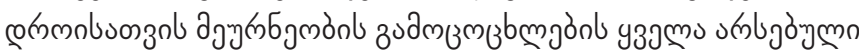

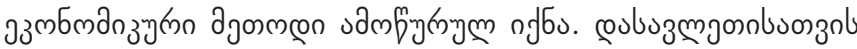

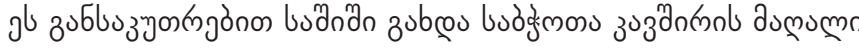

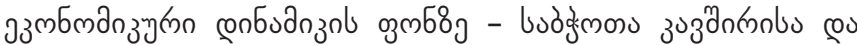

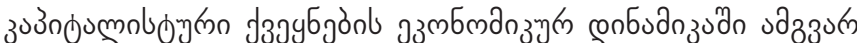

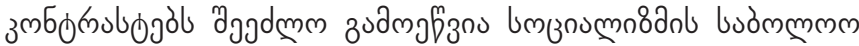

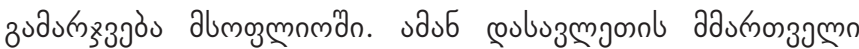

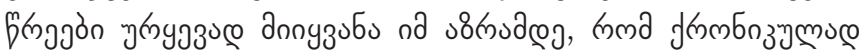

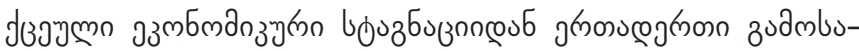

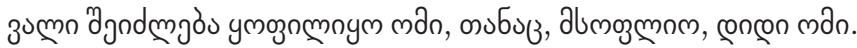

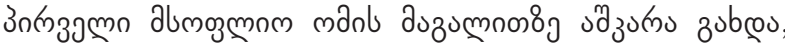

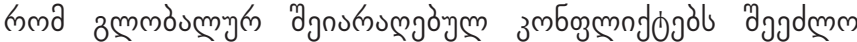

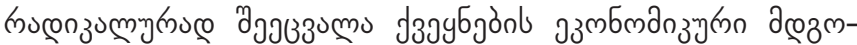

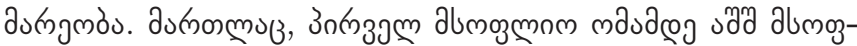

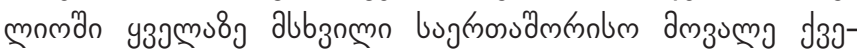

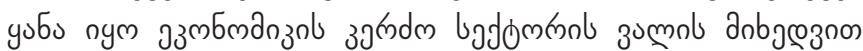

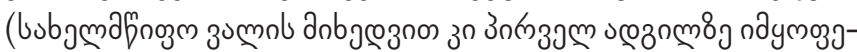

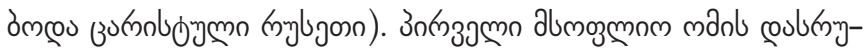

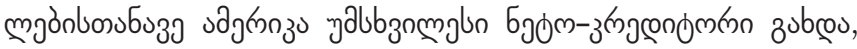

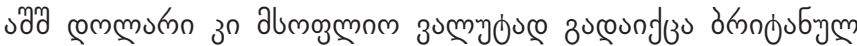

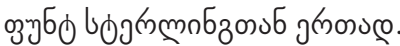

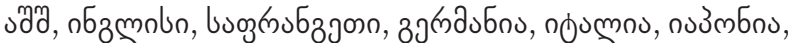

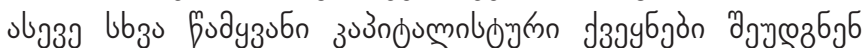

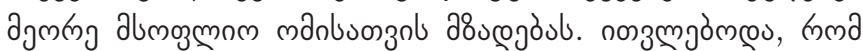

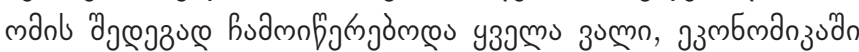

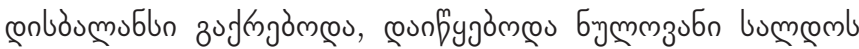

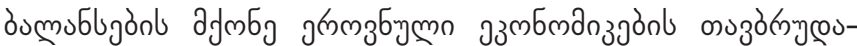

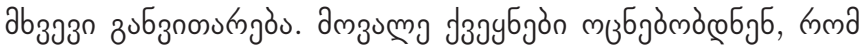

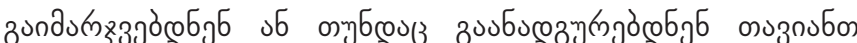

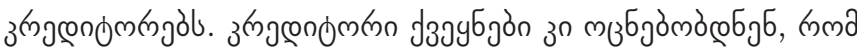

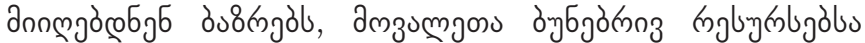

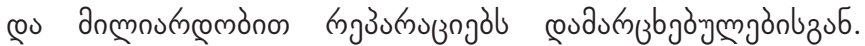

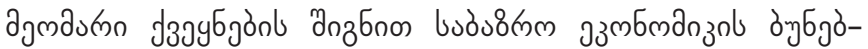

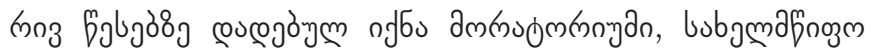

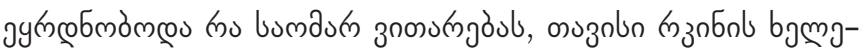

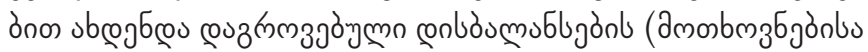

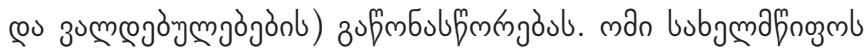

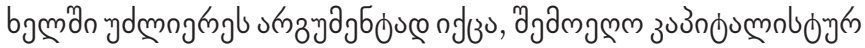

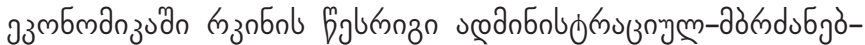

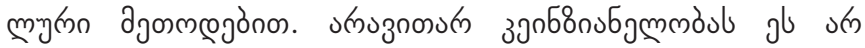

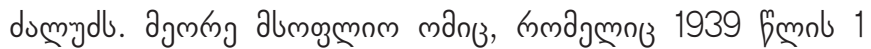

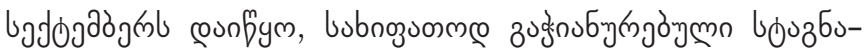

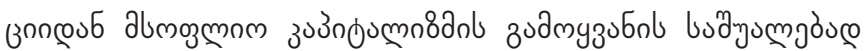
nflos.

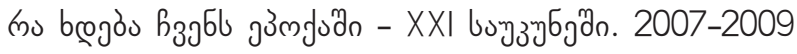

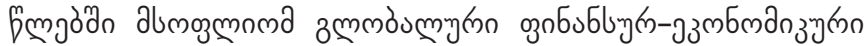

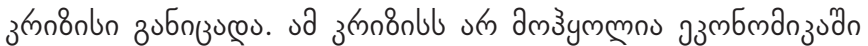

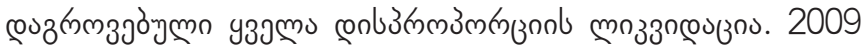

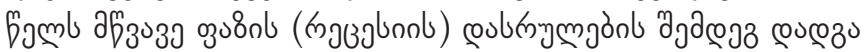

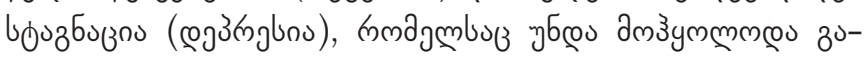

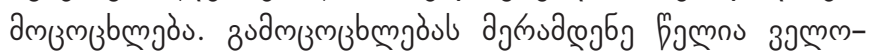

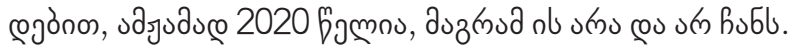

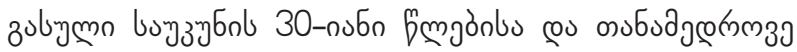

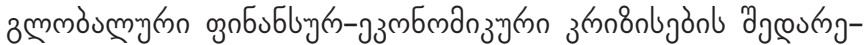

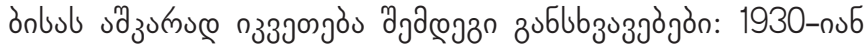

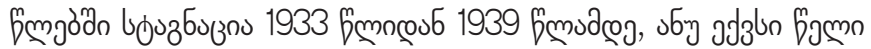

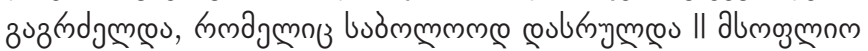

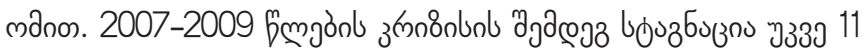

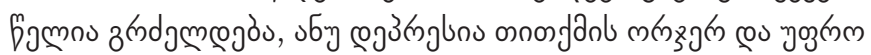

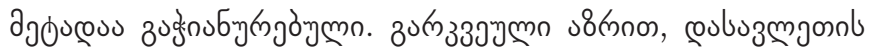

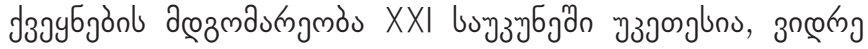

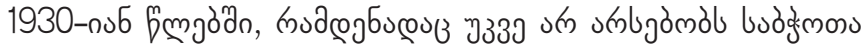

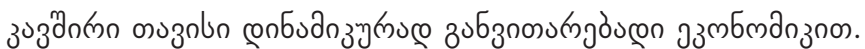

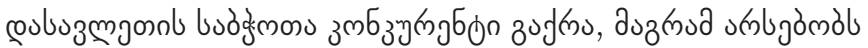

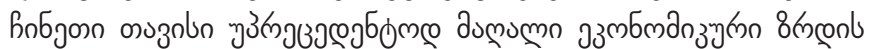

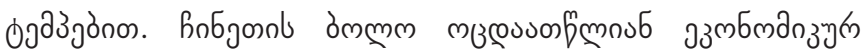

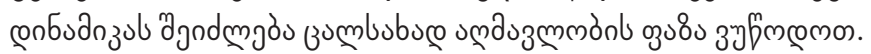

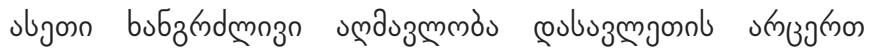

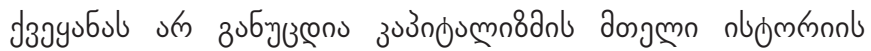

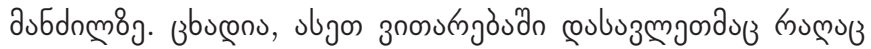

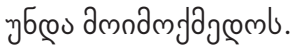

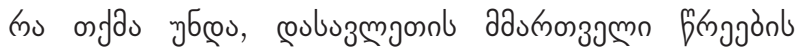

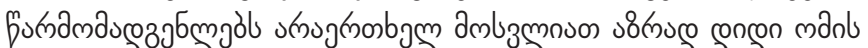

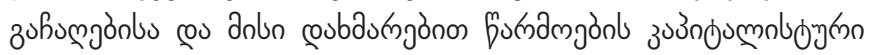

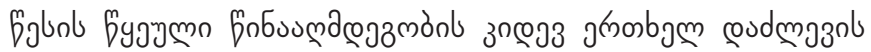

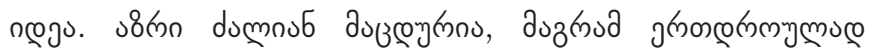

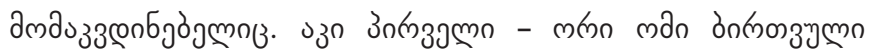

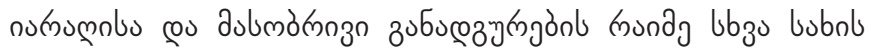

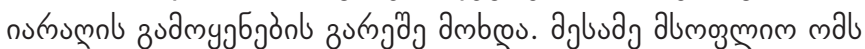

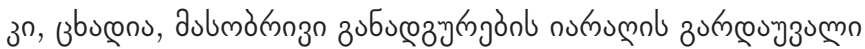




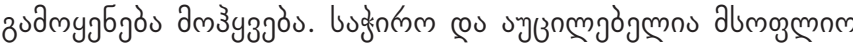

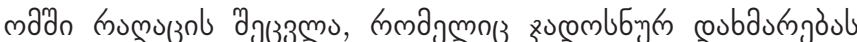

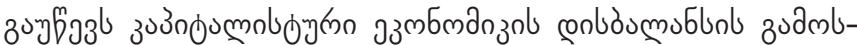

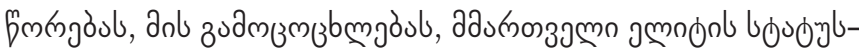

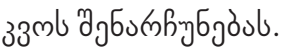

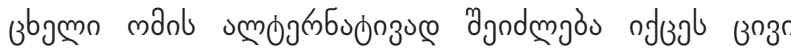

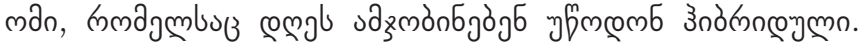

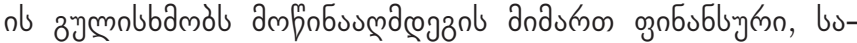

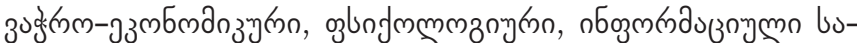

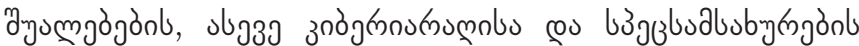

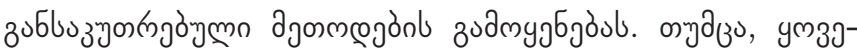

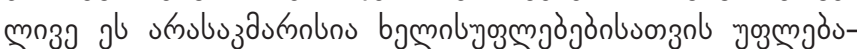

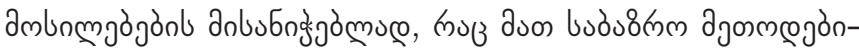

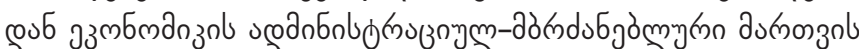

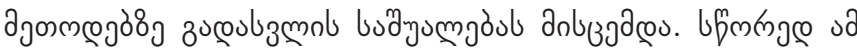

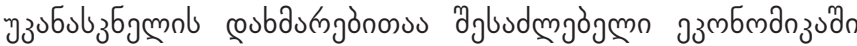

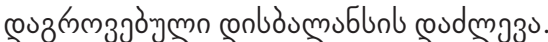

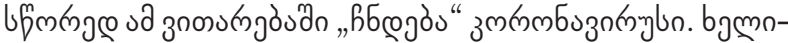

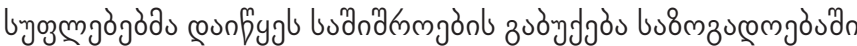

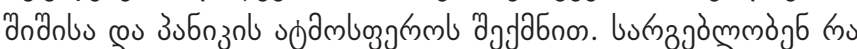

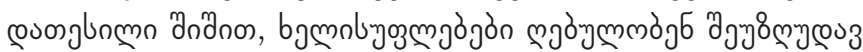

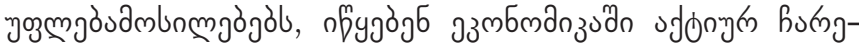

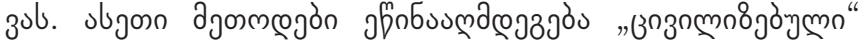

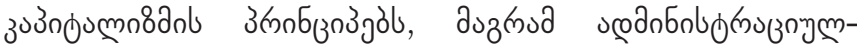

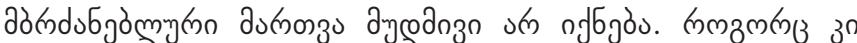

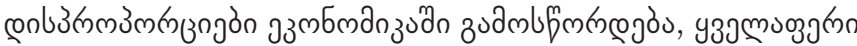

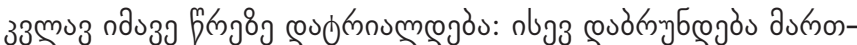

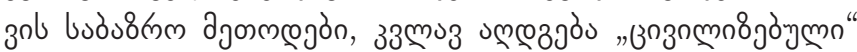

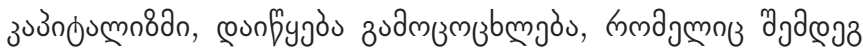

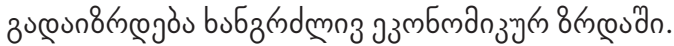

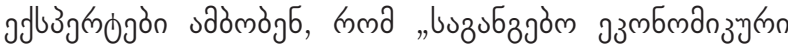

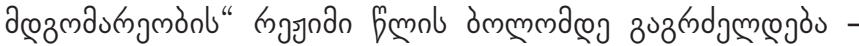

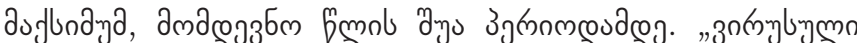

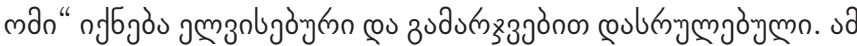

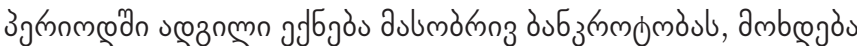

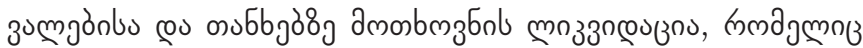

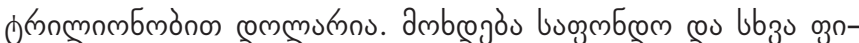

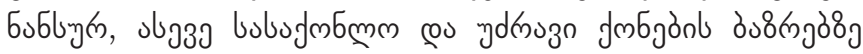

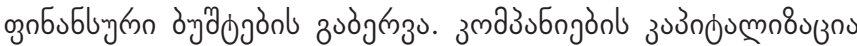

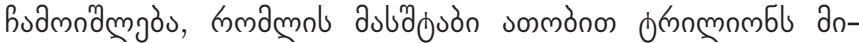

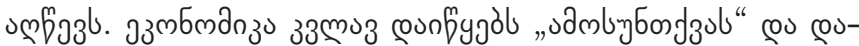

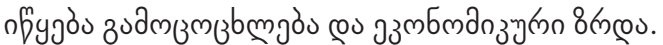

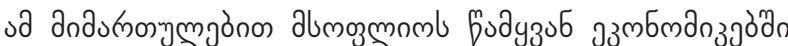

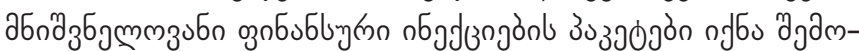

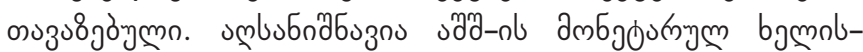

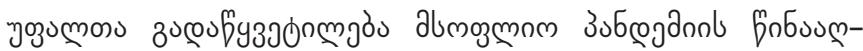

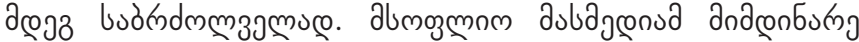

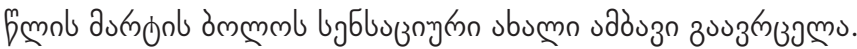

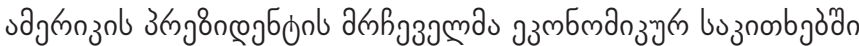

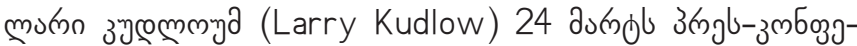

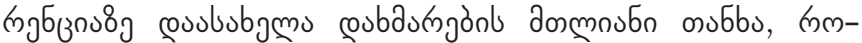
agmbur s ad

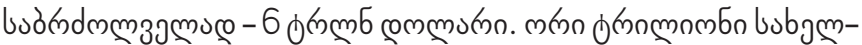

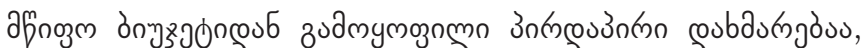

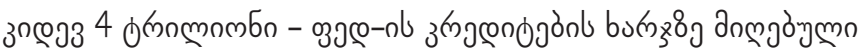
coobbongos (https://www.nationalreview.com/news/ coronavirus-relief-white-house-adviser-larry-kudlowprojects-aid-package-to-reach-roughly-6-trillion/).

adzlo o

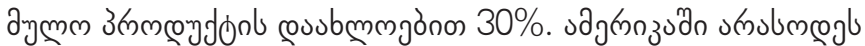

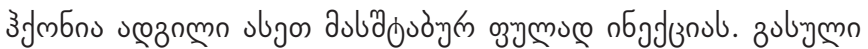
buचzचб

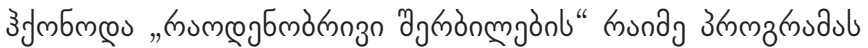

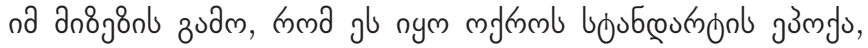

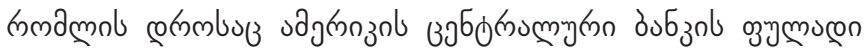

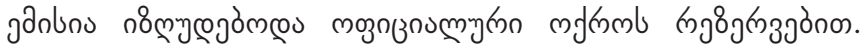

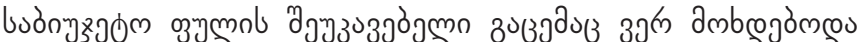

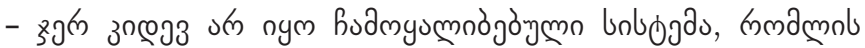

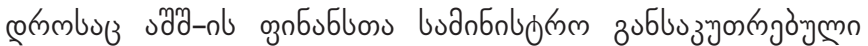

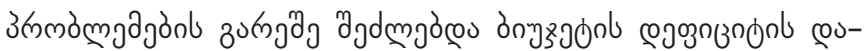

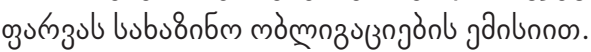

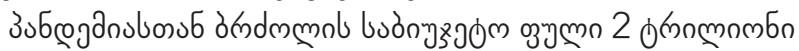

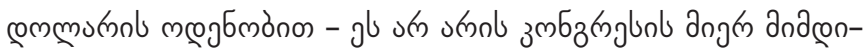

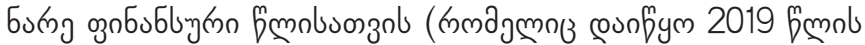

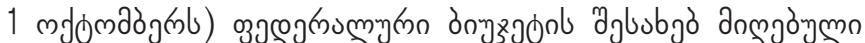

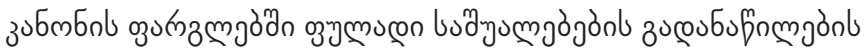

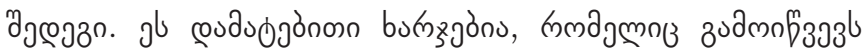

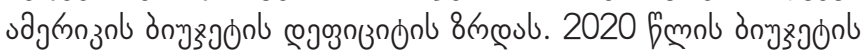

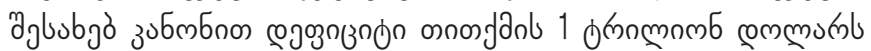

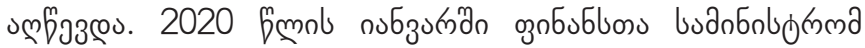

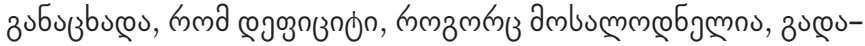

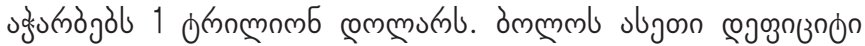

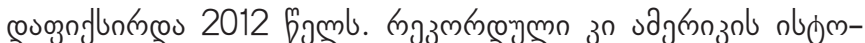

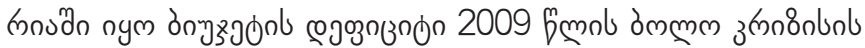

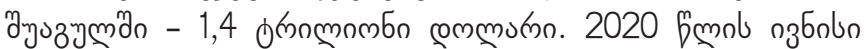

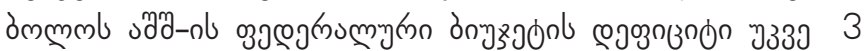

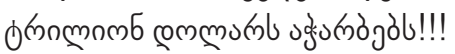

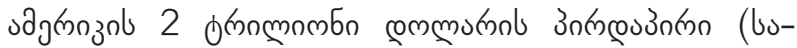

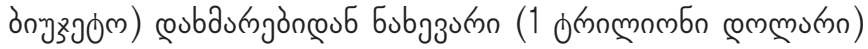

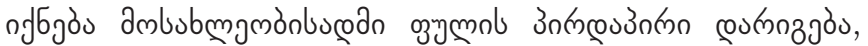

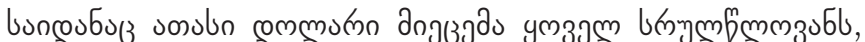

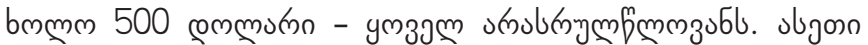

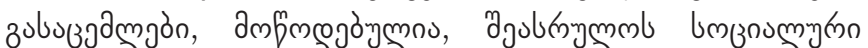

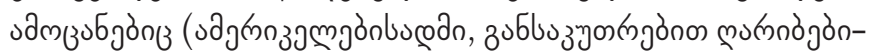

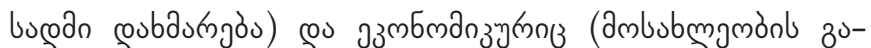

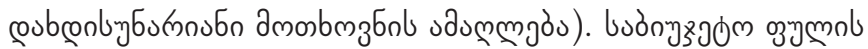

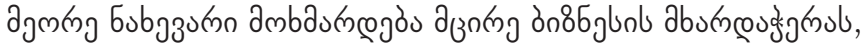

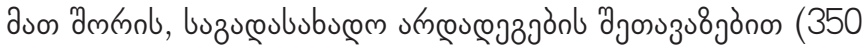

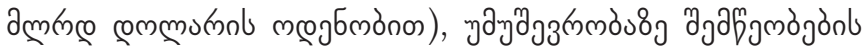

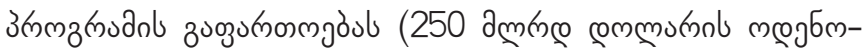




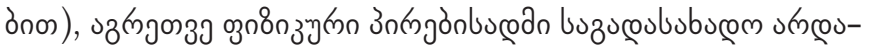

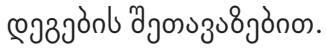

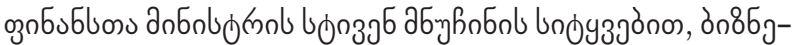

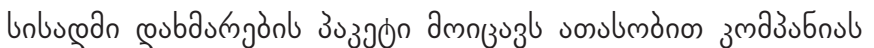

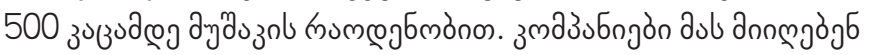

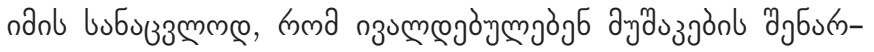

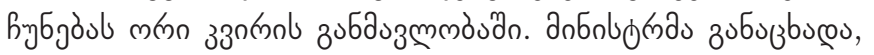

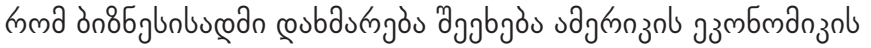

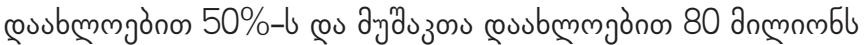

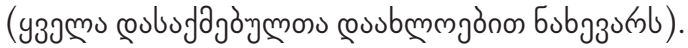

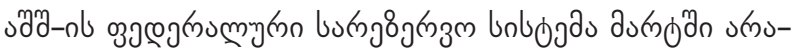

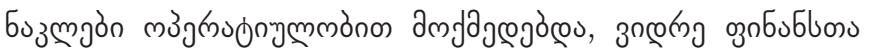

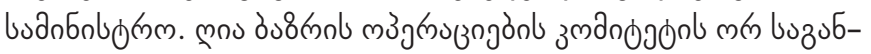

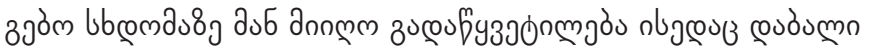

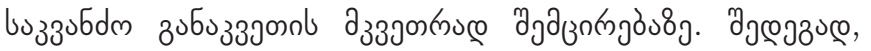

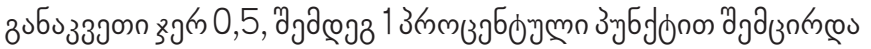

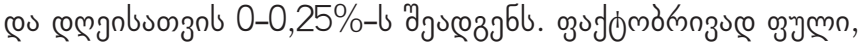

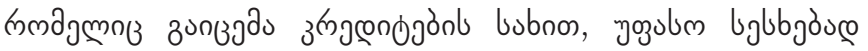

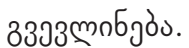

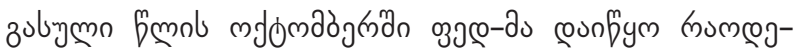

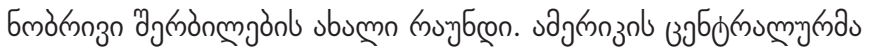

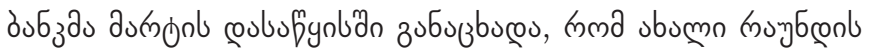
оुu

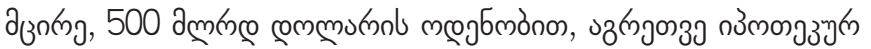

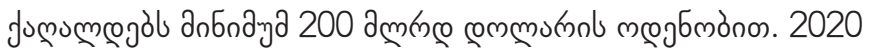

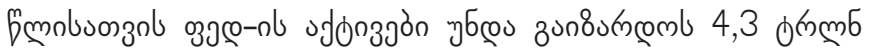

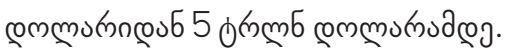

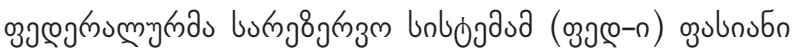

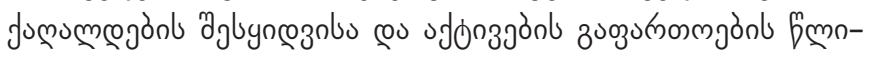

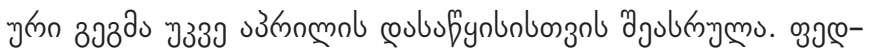

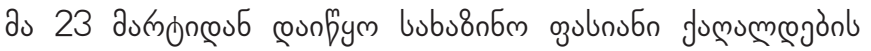

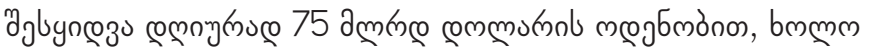

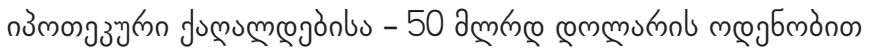

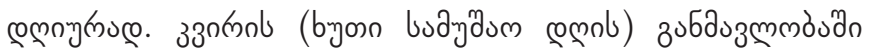

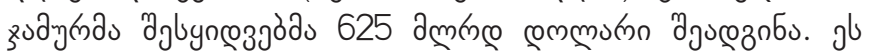

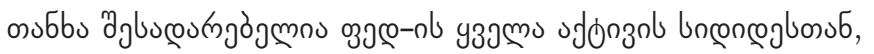

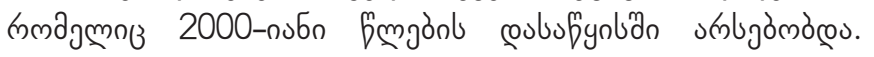

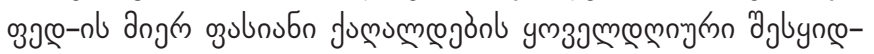

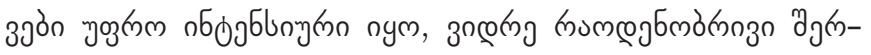

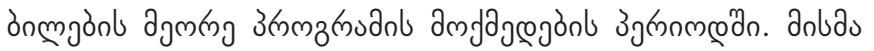

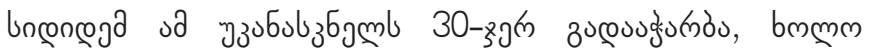

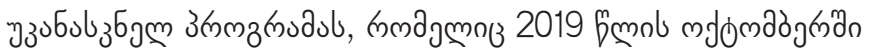

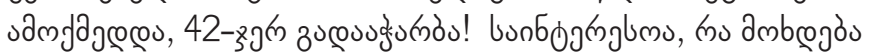

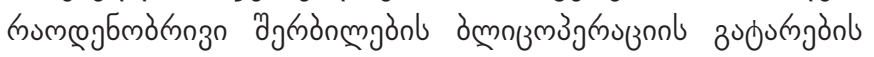

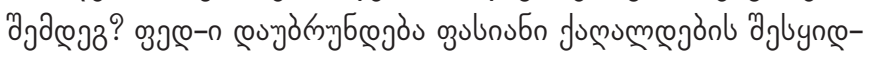

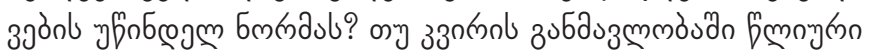

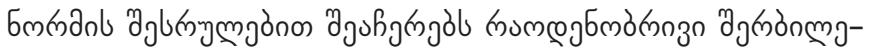

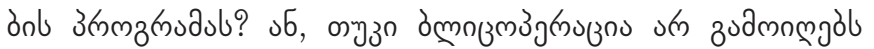

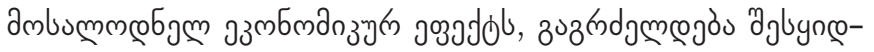

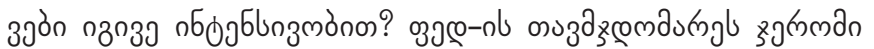

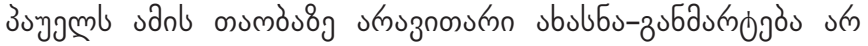

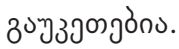

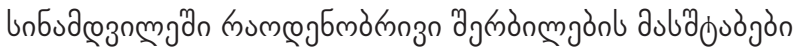

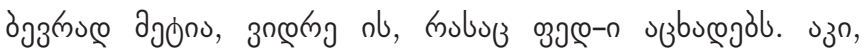

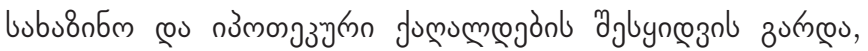

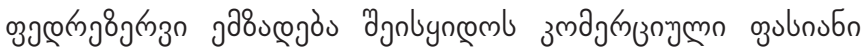

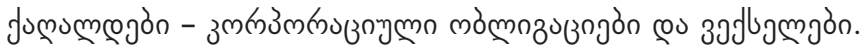

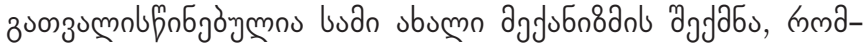

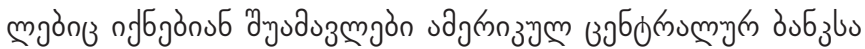

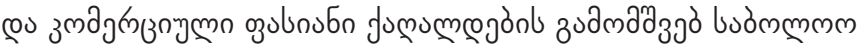

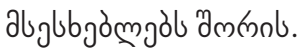

उnగrzamn duon Dmmonl - Primary Market Corporate

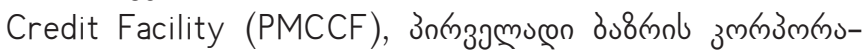

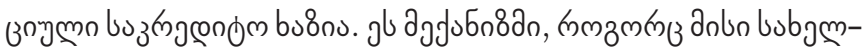

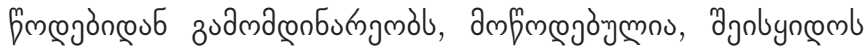

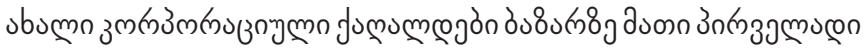
zubonszlyoun comml.

dgmkj - Secondary Market Corporate Credit Faci-

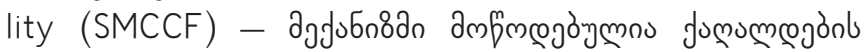

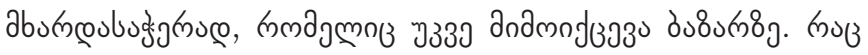

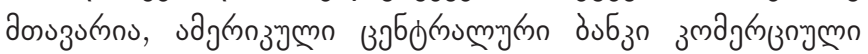

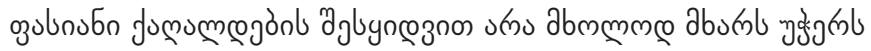

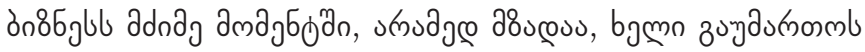

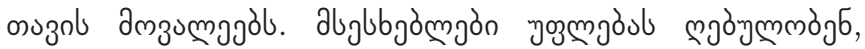

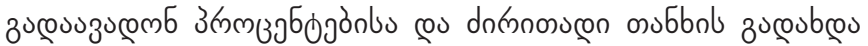

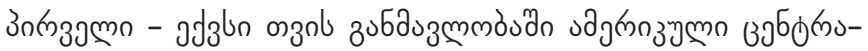

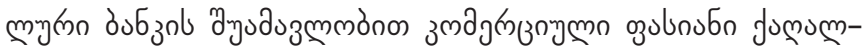

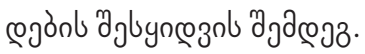

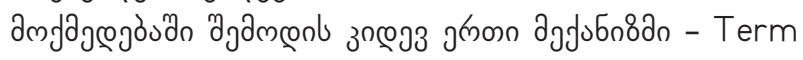

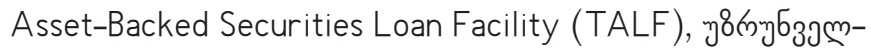

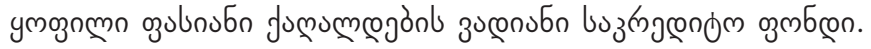

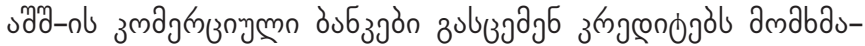

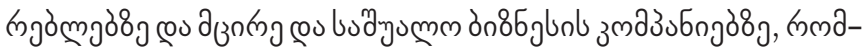

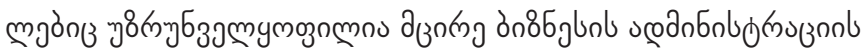

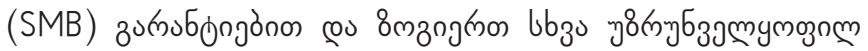

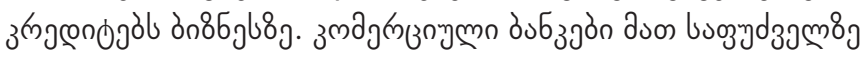

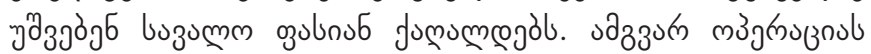

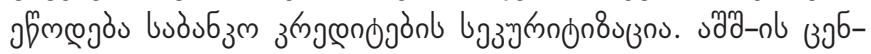

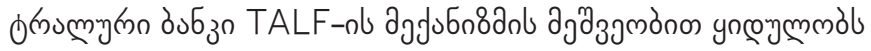

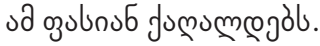

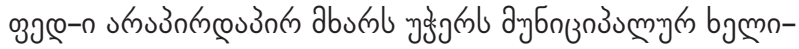

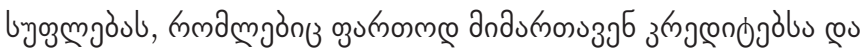

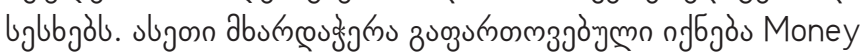
Market Mutual Fund Liquidity Facility (MMLF)-nb oknbjòymn

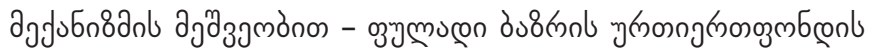

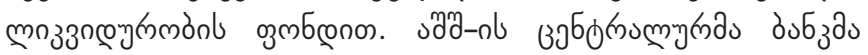

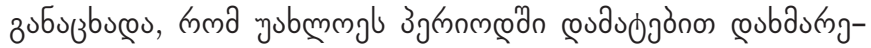

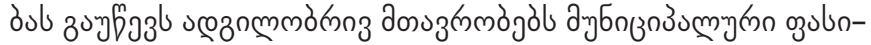

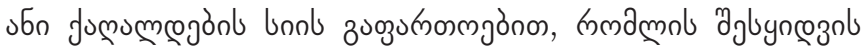

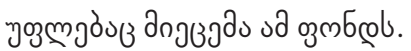




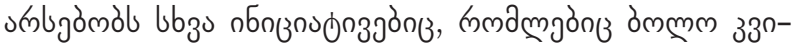

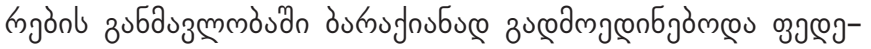

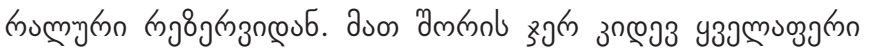

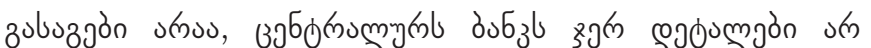

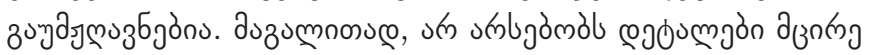

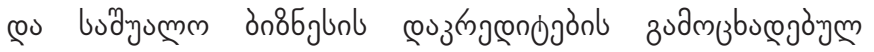

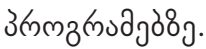

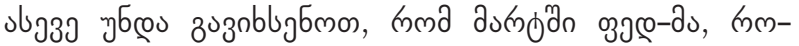

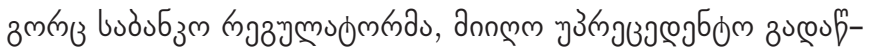

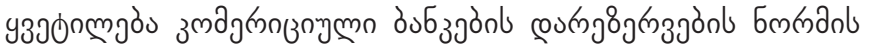

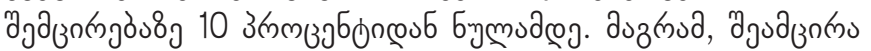

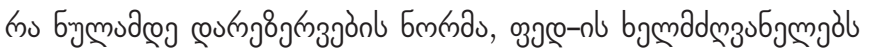

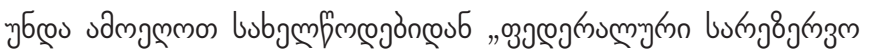

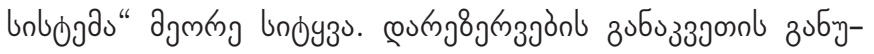

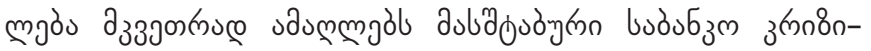

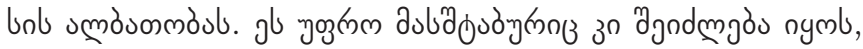
3nم

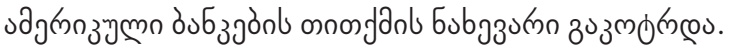

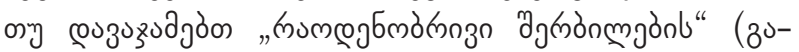

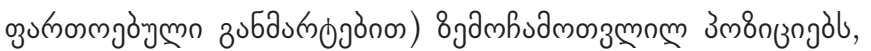
auдn

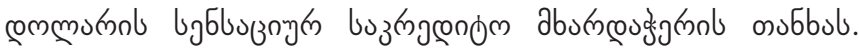

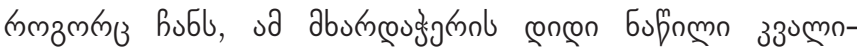

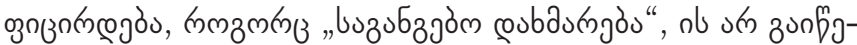

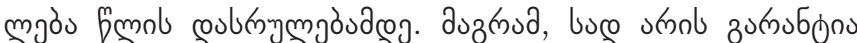

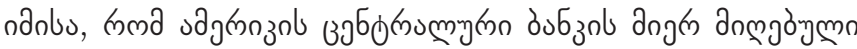

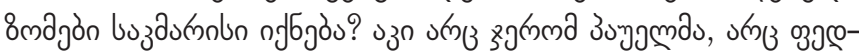

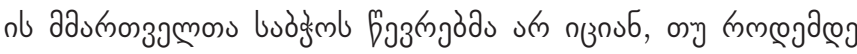

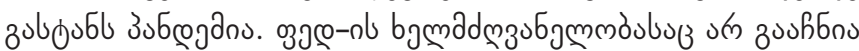

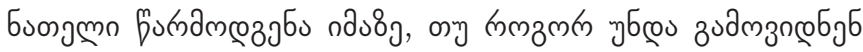

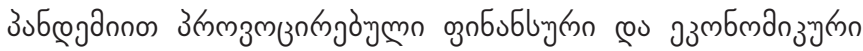

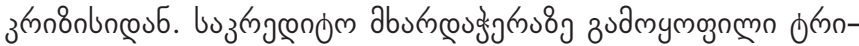

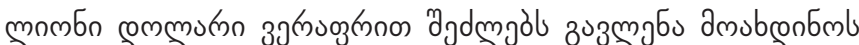

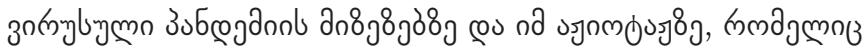
dn

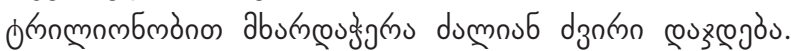

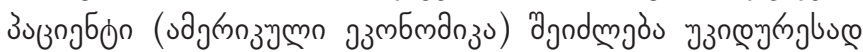
buzumuмm де

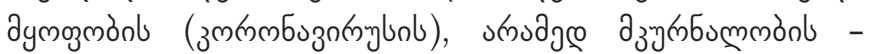

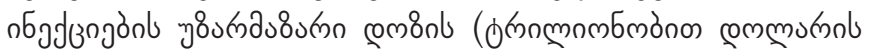

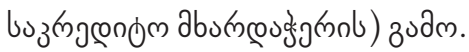

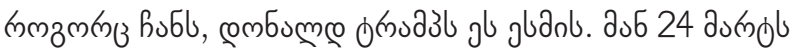

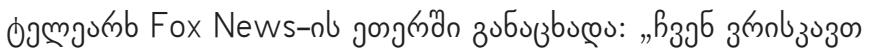

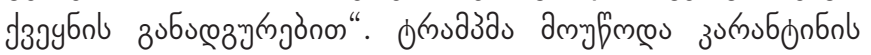

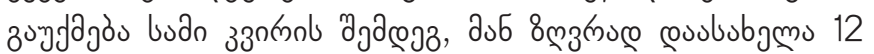

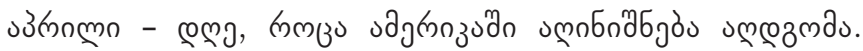

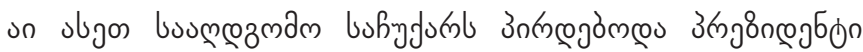

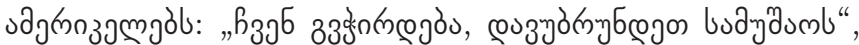

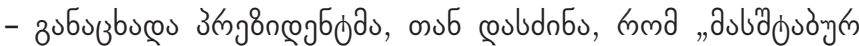

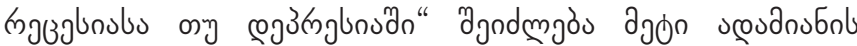

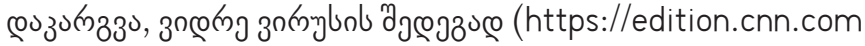
/2020/03/24/politics/trump-easter-economy-coronavirus/ index.html).

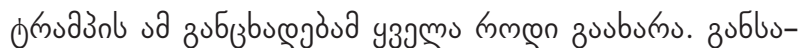

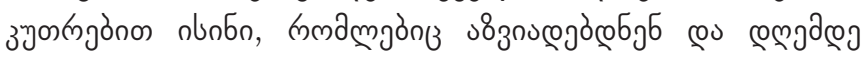

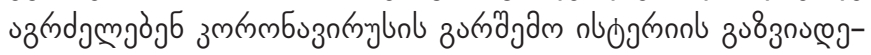

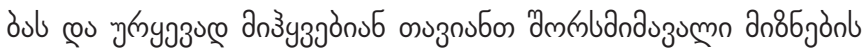

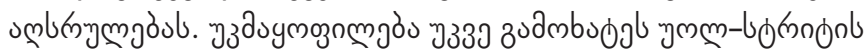

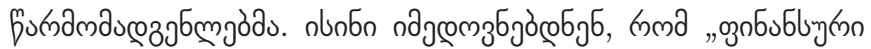

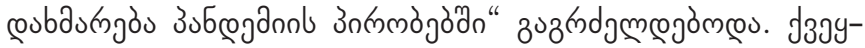

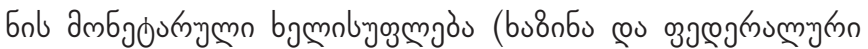

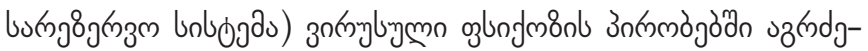

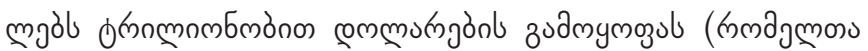

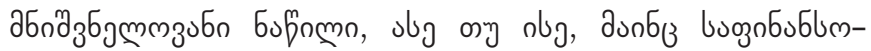

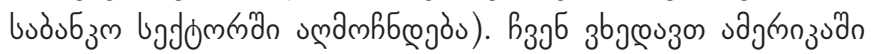

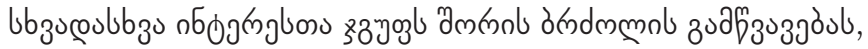

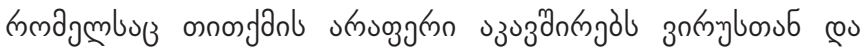
agengnбslions.

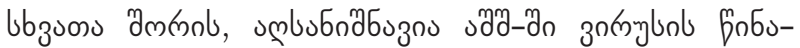

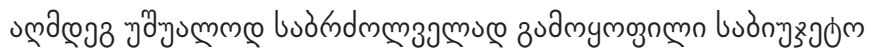

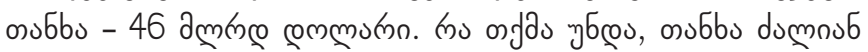

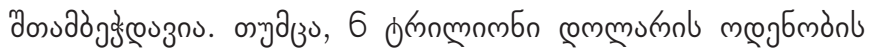

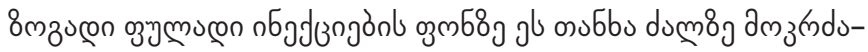

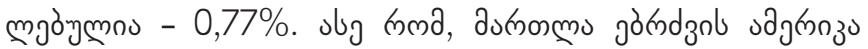

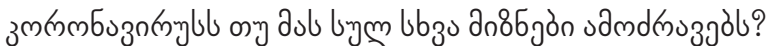

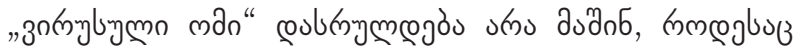

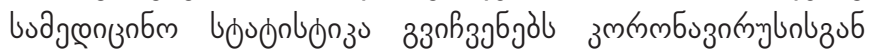

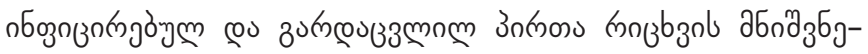

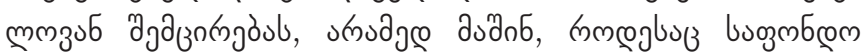

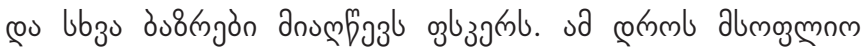

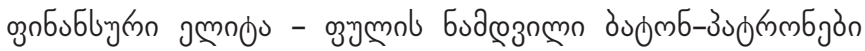

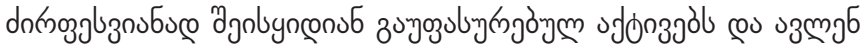

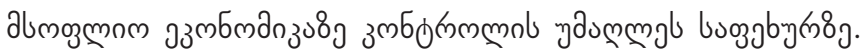

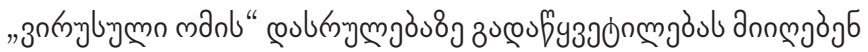

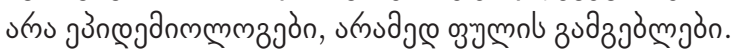

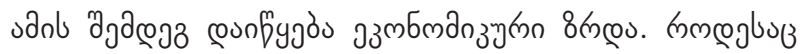

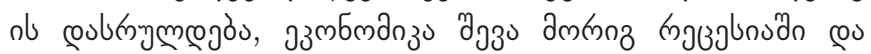

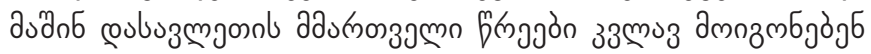

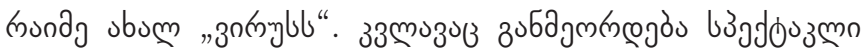

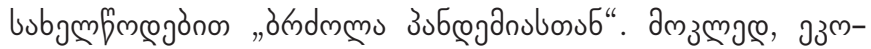

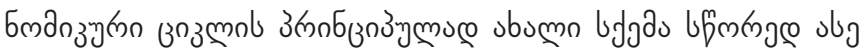

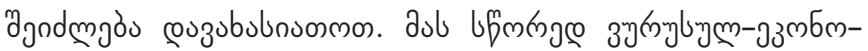

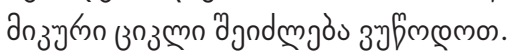

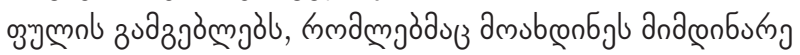
उзбल

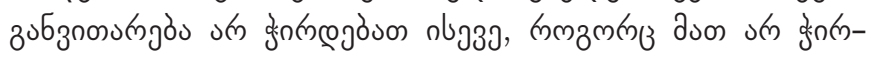

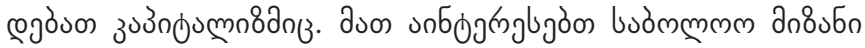

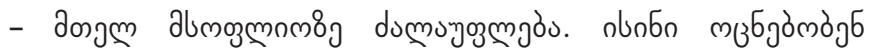

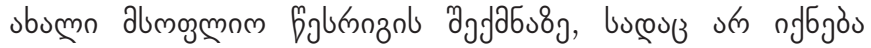

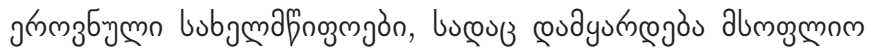




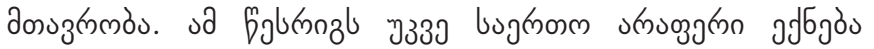

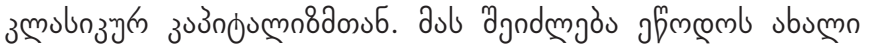

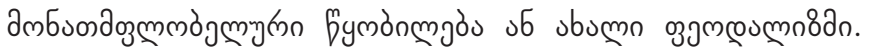

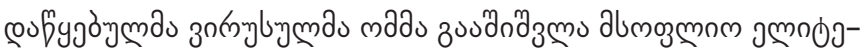

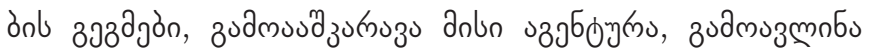

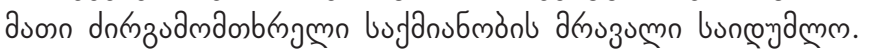
उзбœ

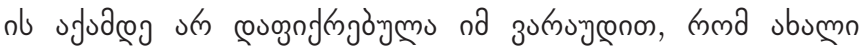

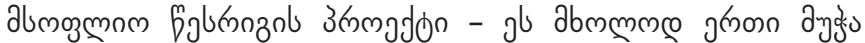

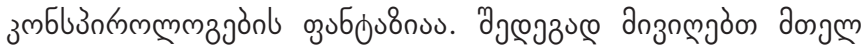

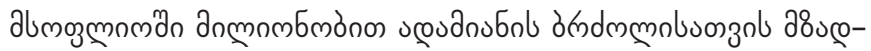

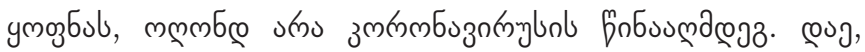

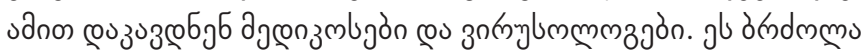

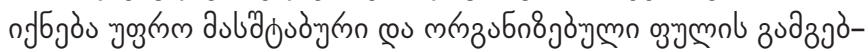

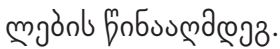

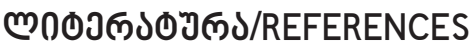

https://www.fondsk.ru/news/2019/10/24/chernyj-chetverg-24-oktjabrja-1929-goda-bankrotstvo-nauchnoobosnovannogo-optimizma-49304.html

https://www.nationalreview.com/news/coronavirus-relief-white-house-adviser-larry-kudlow-projects-aid-package-toreach-roughly-6-trillion/

https://edition.cnn.com/2020/03/24/politics/trump-easter-economy-coronavirus/index.html Tuguell, P. (1957). The Democratic Roosvelt, A Biography of Franklin D. Roosvelt, New York. History of the Second World War (1978). in 12 volumes, vol. 2:341. (In Russian)

https://www.fondsk.ru/news/2019/10/09/anglo-amerikanskij-biznes-s-nacistami-49197.html 